\title{
Mandibular Loop Connector: An Underrated Treatment Modality
}

\author{
${ }^{1}$ Abhijeet R Kore, ${ }^{2}$ Shobha Kore, ${ }^{3}$ Siddharth Gosavi, ${ }^{4}$ Shivsagar Tewari, ${ }^{5}$ Sulekha Gosavi, ${ }^{6}$ Pronob Sanyal
}

\begin{abstract}
Connectors are the components of fixed dental prosthesis that join the individual retainers and pontics together. Connectors may be rigid or nonrigid. Loop connector is a type of rigid connector, indicated primarily when an existing diastema is to be maintained in a planned fixed prosthesis. Such cases can be treated with an implant supported prosthesis or a fixed prosthesis using a loop connector. In case of loop connector, the connector is in the form of a loop on the lingual aspect of the prosthesis that connects the adjacent retainer and pontic. Similarly, loop connector can also be used in the mandibular anterior region for replacement of central incisors, using canines as abutments rather than mandibular laterals which usually have compromised bone support.

There are many reports in literature describing the use of a loop connector in the maxillary anterior sextant. But, there are seldom reports about the use of a loop connector in the mandibular anterior region. This case report describes the use of a mandibular loop connector for the replacement of mandibular teeth where the adjacent teeth are periodontally compromised.
\end{abstract}

Keywords: Connector, Diastema, Fixed prosthesis, Loop connector, Pontic.

How to cite this article: Kore AR, Kore S, Gosavi S, Tewari S, Gosavi S, Sanyal P. Mandibular Loop Connector: An Underrated Treatment Modality. World J Dent 2015;6(3):184-187.

Source of support: Nil

Conflict of interest: None

\section{INTRODUCTION}

Connectors are the components of fixed dental prosthesis that join the individual retainers and pontics together. Connectors may be rigid or nonrigid. Loop connector is indicated primarily when an existing diastema is to be maintained in a planned fixed prosthesis. ${ }^{1}$ Replacement

\section{1,2,4,5Assistant Professor, ${ }^{3,6}$ Professor}

${ }^{1,3-6}$ Department of Prosthodontics, School of Dental Sciences Krishna Institute of Medical Sciences Deemed University, Karad Maharashtra, India

${ }^{2}$ Department of Periodontology, Institute of Medical Sciences and Research, Mayani, Maharashtra, India

Corresponding Author: Abhijeet R Kore, Assistant Professor Department of Prosthodontics, School of Dental Sciences Krishna Institute of Medical Sciences Deemed University, Karad Maharashtra, India, Phone: 08237782452, e-mail: abhijeet. kore@ymail.com of missing teeth in the anterior region is usually a challenging task due to the high esthetic demands of this region. ${ }^{1,2}$ Drifting of teeth into the edentulous area may reduce the available pontic space; whereas a diastema existing before extraction or loss of a tooth may result in excessive mesiodistal pontic space. ${ }^{3,4}$ Such cases can be treated with an implant supported prosthesis or a fixed prosthesis using a loop connector. ${ }^{1,2}$ In these situations, the simplest approach would be to maintain the existing diastema using loop connectors. ${ }^{5,6}$ In case of loop connector, the connector is in the form of a loop on the lingual aspect of the prosthesis that connects the adjacent retainer and/or pontics. ${ }^{3,4}$

There are many reports in literature describing the use of a loop connector in the maxillary anterior sextant. But, there are seldom reports about the use of a loop connector in the mandibular anterior region. This case report describes the use of a mandibular loop connector for the replacement of mandibular teeth where the adjacent teeth are periodontally compromised.

\section{CASE REPORT}

A 22-year-old female patient reported to the Department of Prosthodontics at School of Dental Sciences, Krishna Institute of Medical Sciences Deemed University, Karad, Maharashtra, India, with the chief complaint of missing lower anterior teeth and desired replacement of the same. The patient had undergone extraction of 31 and $41 \frac{11 / 2}{2}$ months ago due to mobility. Clinical examination revealed grade I mobile 32 and 42. Radiographic examination showed almost $50 \%$ bone loss around 32 , 42 and more than $50 \%$ on the mesial proximal aspects. Hence, 32 and 42 were poor candidates to be considered as abutments for a fixed partial denture. Use of lateral incisors and canines on both the sides as abutments was another treatment alternative as it would have provided a splinting effect for the lateral incisors. But, considering the poor bone support, even this option was not considered appropriate as it may have compromised periodontal support of the canines in future. Though placement of implants was a viable alternative, the patient did not agree because of economic factors. She was also reluctant for extraction of 32 and 42 . So, it was finally decided to provide a conventional fixed partial 
denture with the mandibular canines as abutments, to replace both the mandibular central incisors using loop connectors.

\section{PROCEDURE}

Before actually starting the prosthodontic treatment, thorough oral prophylaxis was carried out. Tooth preparation of right and left mandibular canine was done to receive porcelain fused to metal crowns, with an equigingival finish line (Figs 1 and 2). Then gingival retraction was carried out using a medicated retraction cord (Ultrapak E, Ultradent products Inc). A polyvinyl siloxane (Aquasil Soft Putty and Aquasil LV, Dentsply Inti) impression was made using the putty reline technique in a rim-lock impression tray and final cast was obtained in die stone (Kalabhai type IV stone). Another impression was made using alginate ( Neocolloid Zhermack clinical) for fabrication of provisional prosthesis. Temporary prosthesis was cemented on 33 and 43 with noneugenol temporary cement (Provicol, VOCO America) replacing both the central incisors (Figs 3 and 4).

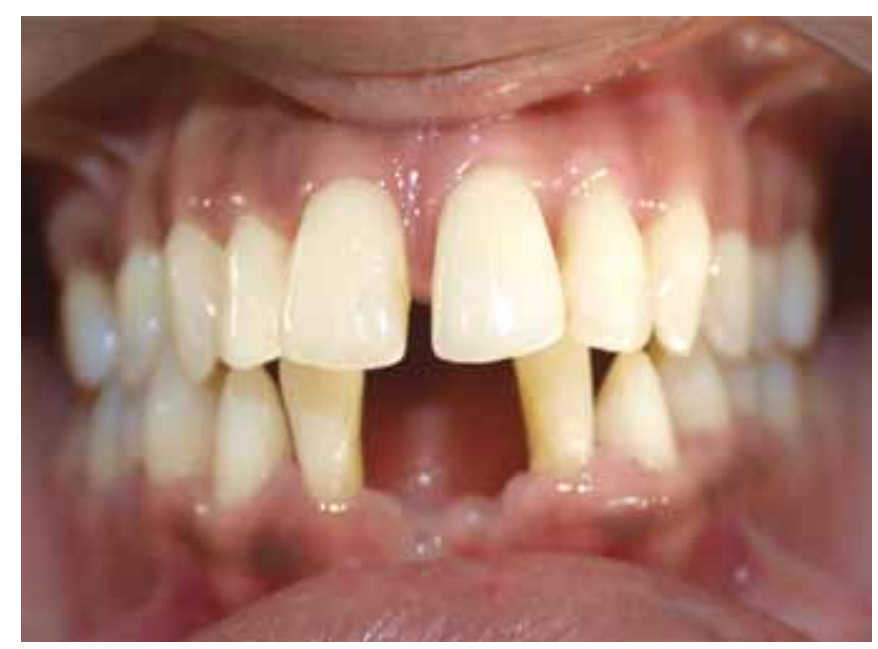

Fig. 1: Abutment preparation: labial view

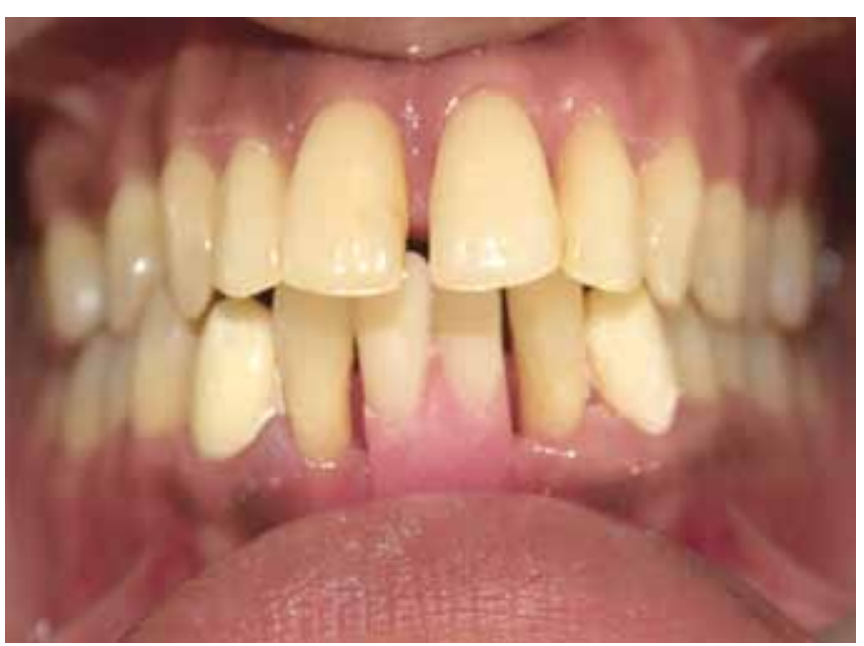

Fig. 3: Provisional: labial view
Two sets of cast were poured from the elastomeric impression, one for preparation of dies while the other was used to get accurate tissue adaptation of the loop. After preparation of dies on 1st cast, wax pattern for the final prosthesis is fabricated. Initially, wax patterns for retainers and pontics are prepared. Then two loops of $2 \mathrm{~mm}$ width; half round in cross-section were fabricated in inlay wax and joined to the retainers' pattern and the pontics' pattern on the lingual aspect. The wax patterns were fabricated with blue inlay wax, casted and copings were finished. Metal try-in was done to verify the fit of the copings as well as of the loop connector. Shade selection was done using Vita 3D master shade guide. Ceramic build-up was completed according to the selected shade (Fig. 5). The final prosthesis was cemented using glass ionomer luting cement (GC, GOLD LABEL, GC Corp, Tokyo, Japan) (Fig. 6). The patient was instructed to maintain proper oral hygiene. Use of dental floss and interdental brush was recommended. Oral hygiene status as well as phonetics was evaluated after 2 weeks. Patient showed satisfactory oral hygiene maintenance and no

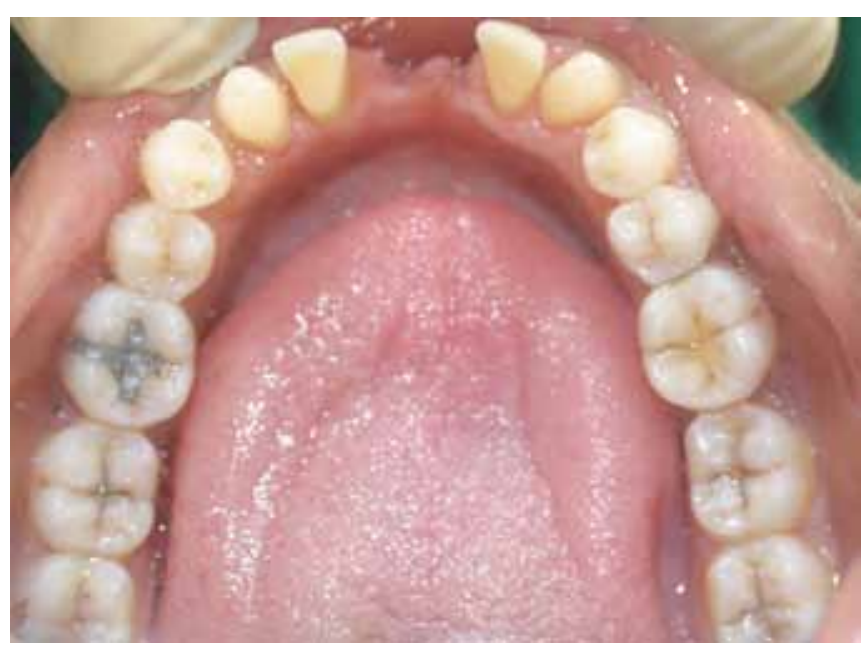

Fig. 2: Abutment preparation: occlusal view

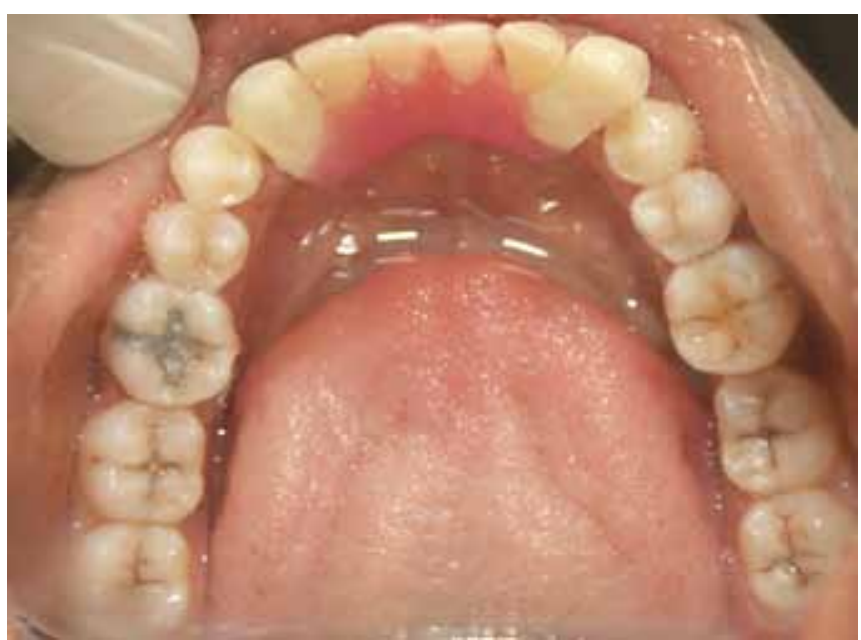

Fig. 4: Provisional: occlusal view 


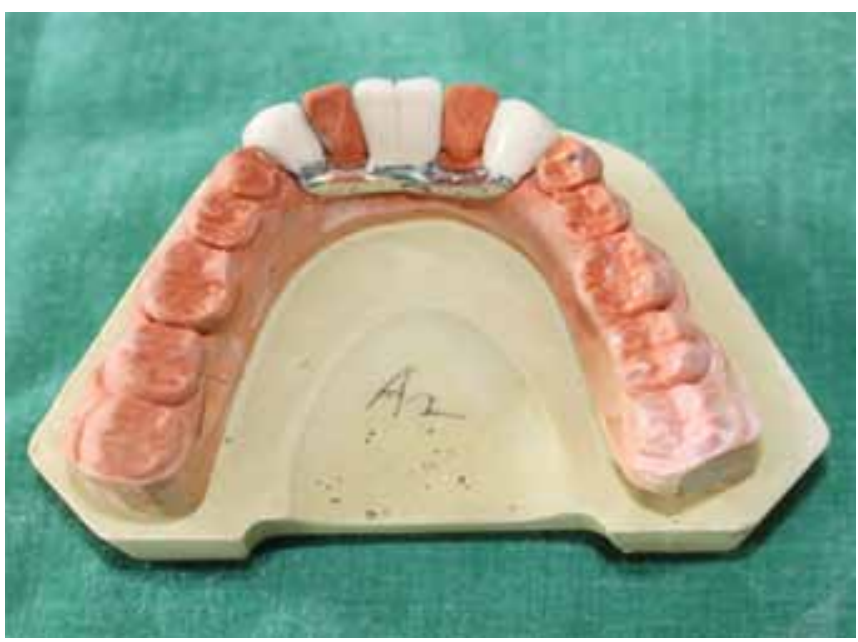

Fig. 5: Final prosthesis on final cast

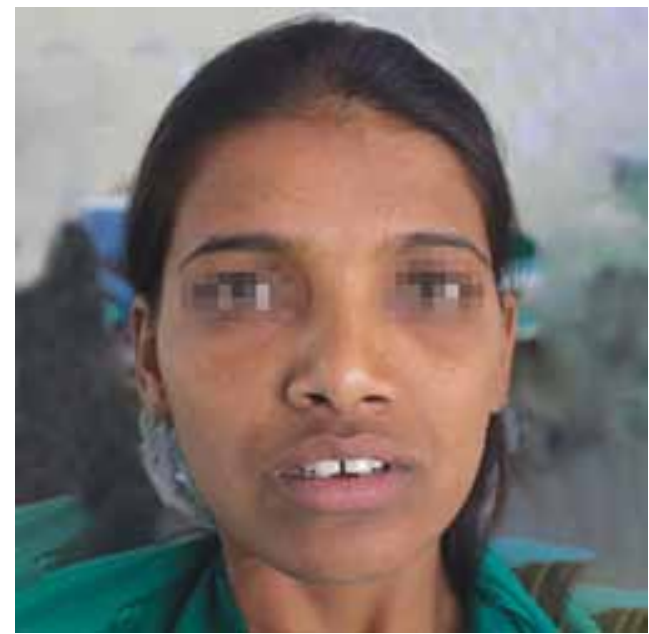

Fig. 7: Preoperative view

difficulty in phonetics. The patient was then instructed to maintain follow-up visits every 6 months. Figures 7 and 8 show the preoperative and postoperative extraoral view of the patient.

\section{DISCUSSION}

Presence of excessive mesiodistal pontic space makes esthetic replacement a great challenge to the dentist. Sometimes the dentist may replace the missing tooth with over contoured retainers and pontics which may lead to unesthetic results. Use of innovative techniques to achieve esthetic results in fixed partial denture treatment procedures makes loop connectors a suitable and a viable treatment option. Maximum esthetic results may be obtained if the natural anatomic forms of the teeth are protected and the diastema are maintained with minimal over contouring of the adjacent teeth. ${ }^{7}$ Use of loop connector in such cases is now well-documented.

At the same time, loop connector also can be used in some other cases when abutment teeth are not in direct vicinity of the edentulous area. We may face such

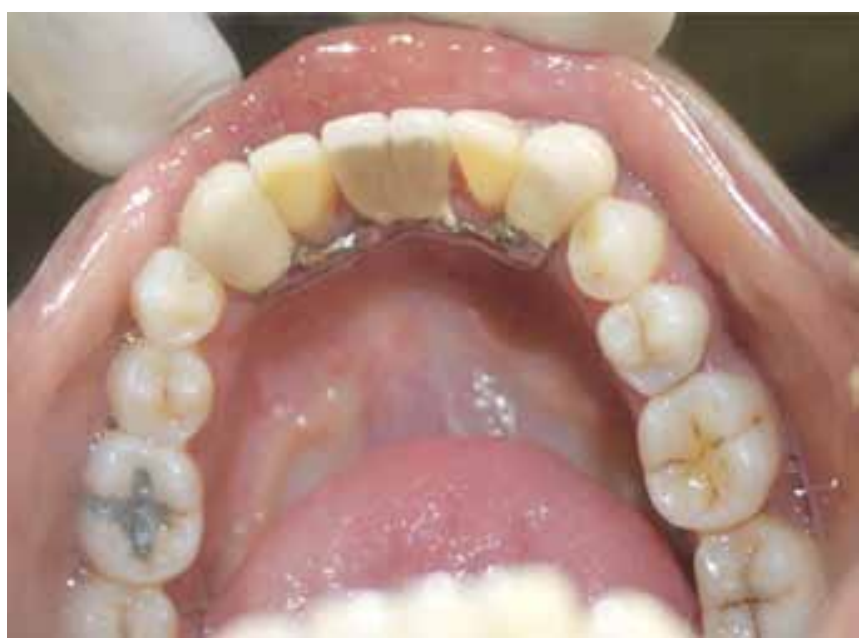

Fig. 6: Final prosthesis after cementation

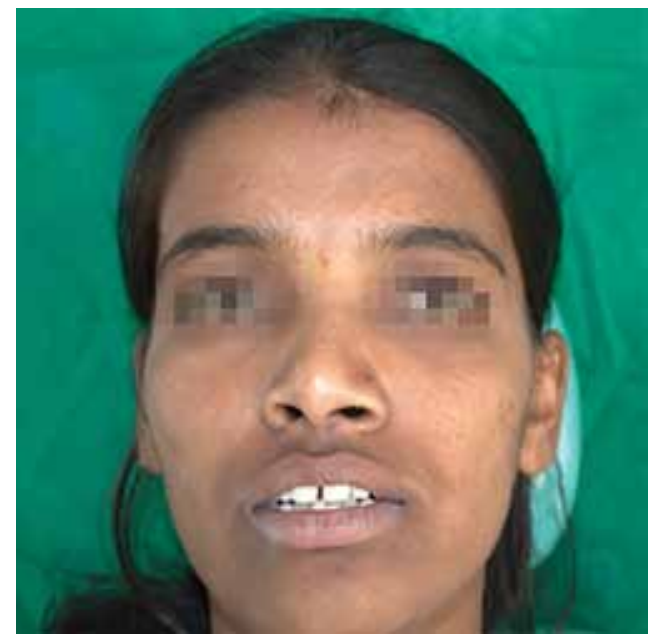

Fig. 8: Postoperative view

condition when the tooth/teeth adjacent to edentulous area are unable to serve as abutment. Though we face such problems regularly in clinical practice, we rarely use loop connector as a solution. Also documentation of such cases in literature is not seen. In the present case report, use of loop connector is described in such a case where mandibular central incisors were replaced with the help of mandibular canines as abutments using loop connector.

Loop connectors have several advantages when it comes to the esthetic appearance, but the patient might object to projecting minor connector in the palatal or lingual region, and it might be a potential food trap for the patient. Meticulous designing is important to ensure that plaque control is not impeded. ${ }^{8}$ While designing loop connector, vestibular depth also should be taken into consideration. Vestibular depth should be sufficient to accommodate loop connector of minimum width required for its rigidity. At the same time, there should be space of at least 3 to $4 \mathrm{~mm}$ between gingival margin and superior margin of loop connector. 
The presence of the palatal loop may make speech uncomfortable in some patients. However, keeping the connector round and small in size will not affect phonetics. Use of loop connectors in mandibular arch as in present case usually does not affect the speech., ${ }^{9,10}$ If the patient can get adapted to the palatally/lingually projecting connector, incorporation of loop connector is an excellent treatment option in cases where excessive space is present in the esthetic zone. ${ }^{11}$

There are many reports in literature that describe maxillary loop connectors in fixed partial dentures. But, there is no report describing the use of loop connectors in the mandibular anterior region. This case involves the use of loop connectors to preserve esthetics in the mandibular anterior sextant. An exceptional challenge in this patient is that the lateral incisors are not periodontally sound to be used as abutments. Hence, this particular treatment design additionally spares the lateral incisors from excessive loading and can be considered as a conservative treatment plan considering the existing clinical condition of the patient.

\section{CONCLUSION}

Various treatment options, like dental implants, resin bonded bridges, conventional fixed partial denture, and cast partial denture, are available to replace missing anterior tooth/teeth. Use of loop connectors help to maintain diastema and uniform spacing between the teeth which enhances the esthetics, especially in case of excessive mesiodistal space in the region of a pontic. At the same time, loop connector also can be used effectively in mandibular arch for replacement of central incisors with the support of mandibular canines as in the present case where lateral incisors were not suitable for the support because of their compromised periodontal condition.

\section{REFERENCES}

1. Rosenstiel S, Land M, Fujimoto J. Connectors for partial fixed dental prosthesis. Contemporary prosthodontics. 4 th ed. New Delhi: Elsevier; 2007. p. 843.

2. Shillingburg HT Jr, Hobo S, Whitsett LD, Jacobi R, Brackett SE. Fundamentals of fixed prosthodontics. 3rd ed. New Delhi: Passi; 2002

3. Tylman S, Malone W, Koth D. Tylman's theory and practice of fixed prosthodontics. Mosby Company; 1978.

4. Puranik S, Kumar P. Anterior spring cantilever fixed partial denture: a simple solution to a complex prosthodontic dilemma. Int J Dental Clinics 2010;2(3):61-63.

5. Kamalakanth S, Arbaz S. Anterior loop connector fixed partial denture: a simple solution to a complex prosthodontic dilemma. J Ind Prosthodontic Society 2008;8(3):162-164.

6. Smith BG. Planning and making crowns and bridges. 3rd ed. London, Martin Dunitz Ltd; 1998. p. 191-206.

7. Bello A, Jarvis RH. A review of esthetic alternatives for the restoration of anterior teeth. J Prosthet Dent 1997;78(5): 437-440.

8. Sharma N, Palekar U, Sharma S. Loop connector-bridging the gaps: a case report. NJDSR 2012 Jan;1(1):42-45.

9. Mitchell D, Mitchell L, Brunton P. Oxford handbook of clinical dentistry. USA: Oxford University Press; 2005.

10. Kalra A, Gowda M, Verma K. Aesthetic rehabilitation of multiple loop connectors. Contemporary Clinical Dentistry 2013 Jan-Mar;4(1):112-115.

11. Madhav VNV, Jadhav R. Treatment options for replacement of missing central incisor. TPDI 2011 Jan;2(1):6-7. 